
\title{
3 Research Square \\ Synthesis, Tribological Properties and Mechanism of Nickel Nanoparticles as Additives in Lithium Grease
}

\section{Hong Zhang}

Shanghai Advanced Research Institute, Chinese Academy of Sciences

wenjing hu

Shanghai Advanced Research Institute, Chinese Academy of Sciences

\section{Jiusheng Li ( $\nabla$ lijs@sari.ac.cn )}

Shanghai Advanced Research Institute, Chinese Academy of Sciences https://orcid.org/0000-00022651-5198

\section{sheng Han}

Shanghai Institute of Technology

\section{Research Article}

Keywords: lithium grease, nickel nanoparticles, tribological performance, lubricating

Posted Date: August 31st, 2021

DOI: https://doi.org/10.21203/rs.3.rs-837241/v1

License: (c) (i) This work is licensed under a Creative Commons Attribution 4.0 International License. Read Full License 


\section{Abstract}

The advanced nano-additives can effectively improve the tribological properties of grease, which can greatly reduce friction consumption. Therefore, we prepared nickel nanoparticles by direct reduction method using $\mathrm{Ni}(\mathrm{HCOO})_{2} \cdot 2 \mathrm{H}_{2} \mathrm{O}$ as the basic raw material. The morphology and structure of the nanoparticles were characterized by scanning electron microscope (SEM), transmission electron microscopy (TEM), X-ray diffraction (XRD), FT-IR spectrometer (FT-IR) and thermal gravimetric analysis (TGA). In order to investigate the lubrication performance of nickel nanoparticles in lithium grease, friction experiments were carried out on four-ball friction tester and TE77 ball-on-plate reciprocating model. Then, the worn surfaces were analyzed by scanning electron microscope and white light interferometry. Meanwhile, the element composition and valence state on friction surfaces were detected by energy dispersive spectrometer and X-ray photoelectron spectroscopy. Based on the experimental results, it was concluded that the nickel nanoparticles can effective improved the tribological properties by interlayer sliding. Moreover, the nickel nanoparticles could promote the formation of friction film on boundary lubrication surface and chemical reaction film between friction pairs. This study could provide a new direction for metal nano-additives to improve the tribological properties of grease.

\section{Introduction}

With the increasing automation of machinery, the energy consumption caused by friction and wear cannot be ignored ${ }^{[1-4]}$. Energy loss due to friction and wear accounts for one-third of total energy consumption each year ${ }^{[5-7]}$ and the energy crisis has become an urgent matter. In order to meet the requirements of mechanical equipment, daily life and environmental protection, many countries are vigorously developing excellent performance lubricants. Extensive research and experience in the past decades have shown that the most effective way to save energy and reduce wear were to apply lubricants, while solid lubrication and liquid lubrication are the main ways to use. Liquid lubrication (mainly oil) has the characteristics of high fluidity and easy to form a stable oil film between friction pairs, while solid lubricant (mainly grease) has better sealing performance and longer service life than the former ${ }^{[8-9]}$. In addition, the solid lubricant can adapt to high temperature, humidity, sea water and other harsh environments, enhance the anti-rust ability, reduce the maintenance time and effective reduce the production cost.

The greases widely used in machinery and equipment include lithium-based grease, polyurea grease, calcium grease and complex grease. Among them, lithium-based greases account for the highest proportion, exceeding $75 \%$ of global production ${ }^{[10-11]}$. The lithium-based grease is widely used in machinery, manufacture and other industrial fields, due to its characteristics of water resistance, rust resistance and oxidation stability [7,12-13]. In recent years, Nano-lubricating additives have become one of the hot topics due to their excellent performance in the fields of electrochemistry, thermodynamics and tribology ${ }^{[1,14-15]}$. The common additives include $\mathrm{MoS}_{2}{ }^{[16-17]}$, graphene ${ }^{[18-19]}$, nano- $\mathrm{TiO}_{2}{ }^{[20-22]}$, and MWCNTs ${ }^{[23]}$, which effectively improve the friction and anti-wear properties of grease products. Besides, 
it is found that metal nanoparticles such as $\mathrm{Ni}$ and Cu nanoparticles form a low shear strength lubricating films on the surface of friction pairs. Compared with traditional organic long-chain additives, the use of metal nanoparticles are less chemical products and simple composition in the friction process and the tribological properties of lubricating grease were greatly improved ${ }^{[24-26]}$.

Nickel, as a strong magnetic metal material, the tribological properties of steel coatings by forming composites with other organic compounds in the field of tribology. For example, Wang ${ }^{[27]}$ et al. reported double-layer Ni-P-Cr composite coating was beneficial to improving mechanical and anticorrosion. The GNP/Ni-based superalloy composite coating was prepared by laser melting deposition. Wang QZ ${ }^{[28]}$ et al. prepared new Ni-Cu alternate coatings (Ni-Cu, $\mathrm{Cu}-\mathrm{Ni})$, nickel and copper coatings with low carbon steel electroplating technology. The results show that $\mathrm{Ni}-\mathrm{Cu}$ coatings have good wear resistance and loading capacity. Song ${ }^{[29]}$ et al. prepared GNP/Ni based superalloy composite coating by laser melting deposition method, and the state of graphene nanoplatelets, friction and wear performance of composite coating were studied. However, nickel nanoparticles can form a good protective film and have a great prospect in the field of lubrication due to their characteristics and crystal structure. Therefore, the tribological properties of nickel nanoparticles as grease additives were further discussed.

In this study, the nickel nanoparticles were synthesized from $\mathrm{Ni}(\mathrm{HCOO})_{2} \cdot 2 \mathrm{H}_{2} \mathrm{O}$ by direct reduction method. Tribological properties of nickel nanoaprticles with different concentrations as additives in lithium grease were studied by two exposure ways. Therefore, the lubricating mechanism of nickel nanoparticles in lithium grease was discussed by analyzing the morphology and valence state of surface chemical elements of wear scars.

\section{Experimental}

\subsection{Materials}

A kind of lithium grease was selected for lubrication testing which was purchased form Hupai Petroleum Co., Ltd., China. $150 \mathrm{~N}$ was used as base oil, the kinematic viscosity of which is $28.008 \mathrm{~mm}^{2} / \mathrm{s}$ at $40^{\circ} \mathrm{C}$ and $5.2033 \mathrm{~mm}^{2} / \mathrm{s}$ at $100^{\circ} \mathrm{C}$. Poly alpha olefins (PAO4), petroleum ether and alcohol were applied directly

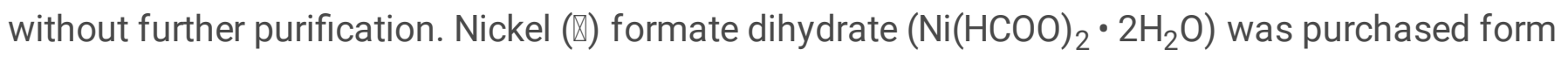
Shanghai Aladdin Co., Ltd.

\subsection{Preparation of Nickel nanoparticles and Lithium Grease}

\subsubsection{Synthesis of Nickel Nanoparticles}

$\mathrm{Ni}(\mathrm{HCOO})_{2} \cdot 2 \mathrm{H}_{2} \mathrm{O}(10 \mathrm{~g}, 54.1 \mathrm{mmol})$ and the oil of PAO4 $(40 \mathrm{~mL})$ were added to the three port round bottom flask and raised temperature to $230^{\circ} \mathrm{C}$ directly. Whole reaction was completed in $\mathrm{N}_{2}$ to ensure the anhydrous and anaerobic environment. After the reaction was cooled to room temperature, the solution 
was separated the oil and nanoparticles through centrifugal under $6000 \mathrm{rpm} / \mathrm{min}$ for $3 \mathrm{~min}$. The obtained black product was washed with petroleum ether and alcohol and weigh after dying at $60^{\circ} \mathrm{C}$ for $5 \mathrm{~h}$.

\subsubsection{Preparation of Lithium Grease with Additives}

According to the previous researches of greases, different concentrations of nickel nanoparticles (nano$\mathrm{Ni}$ ) additives (0, $0.05 \mathrm{wt} . \%, 0.1 \mathrm{wt} . \%, 0.2 \mathrm{wt} . \%, 0.3 \mathrm{wt} . \%)$ contained lithium grease were prepared. The preparation method by the following steps: Firstly, all instruments were dried after washed with petroleum ether and alcohol to ensure no additional impurities. Secondly, nickel nanoparticles were poured into $150 \mathrm{~N}$ oil $(5 \mathrm{~mL})$ and ultrasonic dispersed instrument for $3 \mathrm{~min}$ in order to reduce the aggregation of nanoparticles in grease. Then, the lithium grease $(50 \mathrm{~g})$ and corresponding nickel nanoparticles additives were poured into the beaker and mechanical stirring for $10 \mathrm{~min}$. Lastly, the target greases were obtained after refined grinding by a three-roll grinder for $20 \mathrm{~min}$. In order to eliminate the interference of other factors, the same amount of $150 \mathrm{~N}$ oil was added to the lithium grease without additives.

\subsection{Tribological Test}

The tribological behaviors of nickel nanoparticles used as additives in lithium grease at different concentrations were tested on a four-ball friction tester. The experimental setup and the schematic view of the four-ball assembly of the tribotester was shown in Fig. $1 \mathrm{a}$ and $1 \mathrm{~b}$. The tester was operated with four GCr15 steel balls in total and the lower three balls remain stationary and the upper one is fixed by a clamp. The diameter of the ball is $12.7 \mathrm{~mm}$ and the hardness is HRC 59-61. According to ASTM D4172 method, the wear resistance of grease was evaluated and the conditions were partially modified according to the actual situation. Every test was performed at least three times with a rotating rate of $1200 \mathrm{rpm} / \mathrm{min}$, a load of $196 \mathrm{~N}(1.81 \mathrm{GPa})$, and a temperature of $50^{\circ} \mathrm{C}$ under $60 \mathrm{~min}$ test duration. After the end of the test, the wear scar diameter (WSD) of the three lower balls were measured using a digitalreading optical microscope with an accuracy of $\pm 0.001 \mathrm{~mm}$ and the average WSD was calculated by computer.

In addition, also the friction reducing properties of lithium grease with different concentrations of nickel nanoparticles were evaluated with the TE77 ball-on-plate reciprocating model. The schematic diagram of the experiment was shown in Fig. 1c. The experiment was interrupted when the friction coefficient (COF) reached the value of 0.2 . And $3 \mathrm{~g}$ lithium grease was applied on the surface of the steel plate evenly to obtain a $2 \mathrm{~mm}$ thick layer of grease. When the lower plate was heated to $50^{\circ} \mathrm{C}$, the corresponding contact pressure was $40 \mathrm{~N}(0.87 \mathrm{GPa})$ with the frequency in $3 \mathrm{~Hz}$ and the stroke length was $10 \mathrm{~mm}$. The upper ball specimen was GCr15 steel and the diameter is $10 \mathrm{~mm}$. The lower GCr15 steel plate with a specifications of $58 \mathrm{~mm} \times 38 \mathrm{~mm} \times 4 \mathrm{~mm}$. Each test was repeated at least twice to ensure the accuracy of date. All the balls and plates were washed with petroleum ether and alcohol by ultrasonic vibration cleaners before the tests began. The focus of this study was to observe the tribological performance of the nickel nanoparticles additives and observing WSD and COF values in the rang of boundary lubrication, which was desirable for explore the formation of boundary film or tribofilms. 


\subsection{Characterization}

The structure and morphology of nanoparticles were characterized by scanning electron microscopes (SEM, Tescan Mira 3 XH) and transmission electron microscopy (TEM, Jeol 1011). The infrared spectrum of the target product was measured by fourier transform infrared spectroscopy (FT-IR, Paragon 1000, Perkin Elmer). The crystallinity of the products was analyzed by powder X-ray diffraction (XRD, Smart Lap, Japan) with Cu Ka radiation. The synthesis of nickel nanoparticles were studied accordingly in the rang of $10-90^{\circ}$. The thermogravimetric analyzer (TGA, TA-Q500) evaluated the thermal stability of the nickel nanoparticles under nitrogen, and the flow was performed from 30 to $600^{\circ} \mathrm{C}$ at a heating rate of 10 ${ }^{\circ} \mathrm{C} / \mathrm{min}$. White light interferometry (WLI) was used to observe the wear scar morphology of steel plate. The worn surface morphology of lower steel balls were characterized by SEM. Energy dispersive spectroscopy (EDS, AZtec X-MaxN 80) and X-ray photoelectron spectroscopy (XPS, Thermo-Fischer ESCALAB250Xi) were analyzed the elements between friction pairs, which Al Ka radiation and the binding energies of the target elements were tested at a pass energy of $40 \mathrm{eV}$, and the binding energy of carbon (C1s: $284.60 \mathrm{eV}$ ) was calibrated as reference. XPS provides chemical information of the worn surface and it was used to identify the elements and the composition of the tribofilms.

\section{Results And Discussion}

\subsection{Characterization of nickel nanoparticles}

The morphology and size distribution performance of the prepared nickel nanoparticles were characterized by SEM and TEM. Fig. 2a and $b$ showed that the nickel nanoparticles were obvious spherical morphology, but it is not satisfactory that the nanoparticles have a certain degree of aggregation. Through the measurement and statistics of the particle size of nickel nanoparticles in Fig. 2c. The particle size of nickel nanoparticles were distributed mainly in 130-240 nm and the major particle diameter was approximately distributed in $150 \mathrm{~nm}$ according to the particle size statistics of nickel nanoparticles.

Fig. 3a shows the FTIR spectrum of the prepared nickel nanoparticles. From the vibration of characteristic peaks in the functional group region of infrared spectrum, there are two strong and sharp absorption peaks at $1578 \mathrm{~cm}^{-1}$ and $1340 \mathrm{~cm}^{-1}$, which indicates that the compound contains carbonyl functional groups. As there is no obvious absorption characteristic peak of nickel nanoparticles that it can not be shown in the figure.

The crystal structure of the synthesized nickel nanoparticles was analyzed by XRD. Fig. 3b shows the XRD patterns of the nickel nanoparticles as-prepared. The results show that the different peaks at $44.5^{\circ}$, $51.8^{\circ}$ and $76.4^{\circ}$ correspond to the (111), (220) and (220) crystal planes of nickel. Therefore, it is proved that the synthesized nickel has a face centered cubic (FCC) structure according to the standard card (JCPDS Card No. 04-0850), which indicated that the high purity of the method prepared nickel nanoparticles and there are no other by-products. 
From the TGA curves shown in Fig. 3c, it was found that there are two stages of mass loss. The first stage can be regarded as the loss of water in the air, and the second and third stages are the loss of crystal water of a small part of $\mathrm{Ni}(\mathrm{HCOO})_{2} \cdot 2 \mathrm{H}_{2} \mathrm{O}$ at high temperature, followed by chemical reaction to form nickel, carbon monoxide and carbon dioxide.

\subsection{Friction and wear behavior}

\subsubsection{Anti-wear test}

The anti-wear performance of lithium grease with different concentrations of nickel nanoparticles additives were evaluated by a four-ball friction tester. WSD of the worn surface on the steel ball was shown in Fig. 4. According to the Fig. 4a, the WSD of the grease with nano-Ni declined from $0.607 \mathrm{~mm}$ to rang of $0.426-0.422 \mathrm{~mm}$ before the dosage reached to $0.2 \mathrm{wt} . \%$, decreased by $29.8 \%$ and $30.5 \%$ respectively. Compared with the lithium grease, the WSD of concentration of nano-Ni reached to $0.2 \mathrm{wt} . \%$ decreased by $35.2 \%$. The anti-wear property became worse and the value of WSD was close to $0.1 \mathrm{wt} . \%$ with the further increase of the dosage. The results show that the addition of nickel nanoparticles could significantly improve the anti-wear performance of lithium grease and the anti-friction effect was less sensitive influence when the additive concentration was increased.

The COF curves of the steel balls lubricated by lithium grease with different contents of nickel nanoparticles additives in the friction process is shown in Fig. 4b. The lithium grease without any additives shows high friction coefficient values and instability. After the initial running, the curves of addition of $0.05 \mathrm{wt} . \%$ and $0.1 \mathrm{wt} . \%$ were keep stable and tend to coincide. With the concentration of additives further increased to $0.2 \mathrm{wt} . \%$, the friction coefficient curve was relatively smooth and stability in the whole stage of friction process and average friction coefficient values sharply decreased by $31.8 \%$. However, the $0.3 \mathrm{wt}$ \% with fluctuation in the later friction process after $1800 \mathrm{~s}$.

Based on the above analysis, it can be concluded that the change of additive concentrations can obviously optimize the anti-wear and anti-friction performance. It could be assumed that nickel nanoparticles can effectively reduce wear and helpful form protective film at friction interface.

\subsubsection{Friction coefficient and surface analysis}

The friction response of each of the five blends was determination of instantaneous friction coefficient through spherical contact, every $0.1 \mathrm{~s}$ recording the values for the process of the $30 \mathrm{~min}$ by TE77 reciprocating friction tester. As shown in Fig. 5a, the average COF values of different dosages of nickel nanoparticles additives with lithium grease were lower than the lithium grease. When nickel nanoparticles were added, the friction coefficient decreased from 0.133 to 0.120 and the friction reducing performance was improved by $9.8 \%$. With increasing concentrations of nickel nanoparticles into lithium grease, the change of friction coefficient remains decreasing. The COF decreases from 0.133 to 0.095 with the dosage reaching the optimal value in $0.2 \mathrm{wt} . \%$, the wear resistance increased by $28.6 \%$. With the 
further increase of additive concentration would lead to the deterioration of friction reduction. This may be due to the local bulge caused by the excessive aggregation of nano-Ni on the friction surface.

The coefficient of friction date recorded in-situ during tribological tests at $40 \mathrm{~N}$ as a function of test time was displayed in Fig. 5b. The COF of each of the curves followed similar trends, with the higher staring friction coefficient that reduced during the running-in period, reaching a steady state COF for the remainder of test. The friction curve of lithium grease was broke off and COF values reached over 0.3 after $800 \mathrm{~s}$ sliding, it can be regarded as oil film rupture indicating poor tribological behavior. In contrast, lubricant greases containing nickel produced much lower and remained stable after the running-in period after the first $300 \mathrm{~s}$. The friction curves of nano-Ni remained stable and fluctuated in a small range from 0.109 to 0.114 . The trend of the curve is to keep stable and the $0.2 \mathrm{wt}$.\% nano-Ni is the lowest under the load of $40 \mathrm{~N}$ during the whole test, separated from other concentration curves obviously. With the further increase of additive dosage to $0.3 \mathrm{wt}$.\%, the friction coefficient curve increased which was related to the agglomeration of nanoaprticles. When the nickel nanoparticles enter into the gaps of the friction surface, the van der Waals force is generated between the close nanoparticles to make them aggregate ${ }^{[30]}$.

The white light interferometer is based on the blue light 3D scanning fringe technology to measure the full-scale 3D digital detection of the geometry of the object to be measured. Figure 6 shows the threedimensional morphologies of wear scar surface by lithium grease and contained with different dosages of nickel nanoparticls. It is obvious that there were deep grooves and surface damage on the worn surface when lubricated with lithium grease. When the additive concentration increased to $0.1 \mathrm{wt} . \%$, the depth and height of the wear marks decrease obviously. Under the lubrication condition of lithium grease containing $0.3 \mathrm{wt} . \%$ nano-Ni, the wear marks were narrow and deep, the width of the wear marks were smaller than that of the lithium grease. Therefore, in the reciprocating friction mode, $0.1 \mathrm{wt} . \%$ has the best anti-wear effect. The wear degree increases slightly with the increase of additive concentration. This is similar to the trend of friction coefficient.

According to the above data, it can be concluded that the friction reducing performance of grease with nickel nanoparticles were especially significant compared with the lithium grease. Which indicated that the addition of nickel nanoparticles can effectively improve the strength of oil film. In addition, effective protection was formed between the friction pairs in the friction process so as to enhance the tribological performance of grease.

\subsection{Discussion and analysis of friction mechanism}

\subsubsection{SEM and EDS analysis}

In order to better reveal the lubrication mechanisms of lithium grease with nickel nanoparticles, the morphologies of wear scar surface of steel balls were investigated by SEM and EDS. A typical image of the wear scar formed with two different samples exhibits bright and dark regions. As shown in Fig. 7a, the steel ball with lithium grease presented some deep and wide furrows, irregular abrasions and many broken lines could be observed at the same time. This phenomenon has indicated that the characteristics 
of abrasive wear. SEM image of $0.2 \mathrm{wt}$.\% nano-Ni lubricated wear surface exhibits regions of mild scratches that showed better anti-wear property than the grease. It is further found that smoother surface and lower worn width (Fig. 7b). The results show that the nickel nanoparticles can enter into the friction pairs and effectively promote the tribological performance in the process of friction pairs.

To further complement the finding of the SEM results, The chemical elements of the worn surface were analyzed by EDS. Fig. 8 provide the composition of typical elements on the wear scar after friction process by four-ball friction tester for $60 \mathrm{~min}$. It is shown that there had a large amount of Fe on the surface of all steel balls. Comparing with the surface element of lithium grease, the characteristic elements $\mathrm{Ni}$ could be found on the friction surface lubricated with nickel nanoparticles containing lithium grease. According the element types found in worn surface could be inferred that these nanoparticles can effectively enter the friction pairs from the lithium grease to form a tribofilm, although the surface of steel ball has been washed with petroleum ether before EDS analysis. Those phenomena reflected the chemical reaction during the friction process.

\subsubsection{XPS analysis of the tribofilms}

In order to further analyze the mechanism of lubrication of composite nickel nanoparticles, XPS was used to identify the chemical state of elements through the binding energies of atoms, and the peak fitting was analysis by XPS PEAK software. Fig. 9 depicts high-resolution XPS spectra obtained for C, O, Fe and $\mathrm{Ni}$ elements of wear scar on the GCr15 steel ball lubricated with lithium grease and $0.2 \mathrm{wt} . \%$ nickel nanoparticles as additives in grease at $196 \mathrm{~N}$ for $60 \mathrm{~min}$, respectively. It can be seen that there was obviously peak of Ni element on the worn surface which was consistent with EDS. However, Ni element was not detected on the worn steel surface with lithium grease. Fig. 9A shows the electronic spectrum of lithium grease. In the C1s XPS spectra, the peak of $284.6 \mathrm{eV}$ was attributed to single carbon and $\mathrm{CO}_{2}$ was observed at $286.4 \mathrm{eV}{ }^{[14]}$. The Fe2p3/2 peak at $706.8 \mathrm{eV}, 723.5 \mathrm{eV}$ and $712.8 \mathrm{eV}$ correspond to $\mathrm{Fe}_{3} \mathrm{O}_{4}$ and $\mathrm{Fe}_{2} \mathrm{O}_{3}$, respectively. The $01 \mathrm{~s}$ peak at $530.7 \mathrm{eV}$ which confirmed the formation of $\mathrm{Fe}_{3} \mathrm{O}_{4}$ on the friction surface ${ }^{[31]}$ and the peak appearing at $529.8 \mathrm{eV}$ was attributed to the generation of $\mathrm{Fe}_{2} \mathrm{O}_{3}$. In addition, as one would expect, there was no presence of nickel atoms. Fig. 9B shows the valence analysis of elements on the worn surface with $0.2 \mathrm{wt}$.\% nano-Ni as additives. It can observed the C1s signal has an obvious peak at $290.2 \mathrm{eV}$, most of carbon comes from air in this part. In the Ni2p XPS spectra, Ni 2p3/2 peak at $852.70 \mathrm{eV}$ and the Ni 2p1/2 peak at $869.9 \mathrm{eV}$ indicated that nickel can be released from the grease and transferred onto sliding surface during the friction process. Furthermore, the nickel react with oxygen can be observed to form $\mathrm{Ni}_{2} \mathrm{O}_{3}$ and $\mathrm{NiO}$ (the peaks are located at $856.0 \mathrm{eV}$ and $853.9 \mathrm{eV}$ ). The formation of nickel oxide was also demonstrated in the oxygen spectrum (the $01 \mathrm{~s}$ peaks at $531.5 \mathrm{eV}$ and 529.4 eV) ${ }^{[32]}$.

Base on the analysis of XPS spectra and a comparision of the worn surfaces (see Fig. 7, 8), in the lithium grease regime, the steel ball makes contact directly with the oil film and caused severely scratched. Furthermore, the cracked new worn surface shows a high surface energy and iron atom changed by 
chemical react, $\mathrm{Fe}_{2} \mathrm{O}_{3}$ and $\mathrm{Fe}_{3} \mathrm{O}_{4}$ are the main components of the chemical reaction. For lubricated with 0.2 wt.\% nano-Ni, except for the base reaction with iron, meanwhile, the $\mathrm{Ni}, \mathrm{NiO}$ and $\mathrm{Ni}_{2} \mathrm{O}_{3}$ also formed and filled surface gaps in the contact surface. We can inferred that the nickel nanoparticles can be easily adsorbed on the worn surfaces and contained in the lithium grease formed a boundary lubrication film on the friction pairs. Therefore, the deposition of nickel nanoparticles and the formation of tribochemical reaction products on the worn surface are the effective reasons to improve the tribological properties of lithium grease in the friction process.

\subsubsection{Analysis of the lubricating mechanism}

Based on the above analysis of experimental results, the lubrication mechanism of nickel nanoaprticles as additives in lithium grease was discussed and the schematic diagram was shown in Fig. 10. When the friction pairs are neat lithium grease (Fig. 10a), the effective oil film protection can not be formed between the contact surfaces under continuous pressure and shearing. The abrasive wear and adhesive wear were occurred between steel surfaces ${ }^{[33]}$. When the friction pairs were lubricated by lithium grease with nickel nanoparticles (Fig. 10b), the nickel nanoparticles enter into the friction interface and deposit on the uneven surface and form physical adsorption to make the surface smooth, which reduces the shear force between the friction pairs and further improves the anti-wear ability of the grease. At the same time, due to the structural characteristics of nickel nanoparticles, there was a slipping effect in the lubrication process, so that there were no excessive contact between the steels ${ }^{[34]}$. With the progress of friction (Fig. 10c), chemical reaction takes place on the friction surface to form the tribo-film, which was composed of $\mathrm{Fe}_{2} \mathrm{O}_{3}, \mathrm{Fe}_{3} \mathrm{O}_{4}, \mathrm{NiO}, \mathrm{Ni}_{2} \mathrm{O}_{3}$ and other substances. The addition of nanoparticles can effectively prevent the friction surface from serious wear, and the tribo-film can protect the friction surface, which can improving the tribological properties of lithium grease.

\section{Conclusion}

In summary, the nickel nanoparticles had been successfully prepared via a handy and speedy chemical reaction route and evaluated as tribological additives in lithium grease under steel -steel contact. The tribological performance and lubrication mechanism were studied through various analysis and characterization methods. The results showed that nano-Ni can markedly improve the tribology properties of lithium grease. Especially, the friction reducing performance was improved by $28.6 \%$ and the wear spot diameter decreased by $35.2 \%$ at the optimal concentrations $0.2 \mathrm{wt}$.\%. The worn surface of steel ball lubricated by nano-Ni had fewer shallow furrows and more smooth compared with lithium grease can be observed under the SEM and WLI. By analyzing the morphology and element composition of the worn surface, the lubrication mechanism of nickel nanoparticles as additives were revealed. In lithium grease, the nickel nanoparticles were first adsorbed on the surface of the friction pairs due to their ferromagnetism and slide between layers. After then, the tribo-film was formed by tribochemical reaction, which were mainly composed by $\mathrm{Fe}_{2} \mathrm{O}_{3}, \mathrm{Fe}_{3} \mathrm{O}_{4}, \mathrm{NiO}, \mathrm{Ni}_{2} \mathrm{O}_{3}$ and other inorganic compounds, can 
effectively reduce friction and severe wear. This may provide a reference for the diversity of grease additives.

\section{Declarations}

Funding: This study was funded by "Transformational Technologies for Clean Energy and Demonstration (XDA 21021202)", "YouthInnovation Promotion Association, Chinese Academy of Sciences (2019288)" and "Shanghai Pudong New Area Science and Technology Development Foundation (PKJ2019-C01)" .

Conflicts of interest: The authors declare they have no financial interests.

Availability of data and material: All data and materials meet the standards and ensure their authenticity.

Code availability: Not applicable.

Authors' contributions: All authors contributed to the study conception and design. Material preparation, data collection and analysis were performed by Hong Zhang. The first draft of the manuscript was written by Hong Zhang and all authors commented on previous versions of the manuscript. All authors read and approved the final manuscript.

Ethics approval: Not applicable.

Consent to participate: Not applicable.

Consent for publication: Not applicable.

\section{References}

1. Maksimova Yu. M., Shakhmatova A. S., Ilyin S. O., et al. Rheological and Tribological Properties of Lubricating Greases Based on Esters and Polyurea Thickeners. Rheological and Tribological Properties 2018;58(12):1064-1069.

2. Ye $X Y, W a n g ~ J Q$ and Fan MJ. Evaluating tribological properties of the stearic acid-based organic nanomaterials as additives for aqueous lubricants. Tribology International 2019;140:105848.

3. Wang HD, Wang Y, Liu YH, et al. Tribological behavior of layered double hydroxides with various chemical compositions and morphologies as grease additives. Friction 2020.

4. He Q, Li AL, Guo YC, et al. Tribological properties of nanometer cerium oxide as additives in lithium grease. Journal of Rare Earths 2018;36:209-214.

5. Bai PP, Li SW, Tao DS, et al. Tribological properties of liquid-metal galinstan as novel additive in lithium grease. Tribology International 2018;128:181-189.

6. Zhao JH, Yang GB, Zhang CL, Zhang YJ, et al. Synthesis of water-soluble Cu nanoparticles and evaluation of their tribological properties and thermal conductivity as a water-based additive. Friction 
2019;7(3):246-259.

7. Guo LH, Zhang YM, Zhang G, Wang QH, Wang TM. MXene-Al2O3 synergize to reduce friction and wear on epoxy-steel contacts lubricated with ultra-low sulfur diesel. Tribology International 2021;153:106588.

8. Li ZJ, He Q, Du SM, Zhang YZ. Effect of few layer graphene additive on the tribological properties of lithium grease. Lubrication Science 2020;32:333-343.

9. Zhang RC, Liu XQ, Guo ZG, et al. Effective suger-derived organic gelator for three different types of Iubricant oils to improve tribological performance. Friction 2020;8(6):1025-1038.

10. Shu J, Harris K, Munavirov B, Westbroek R, Leckner J. Tribology of polypropylene and Li-complex greases with ZDDP and MoDTC additives. Tribology International 2018;118:189-195.

11. Niu M, Qu JJ, Gu L. Synthesis of titanium complex grease and effects of graphene on its tribological properties. Tribology International 2019;140:105815.

12. Krawiec S. On the mechanism of the synergistic effect of PTFE and copper in a lithium grease lubricant. Industrial Lubr Tribol. 2011;63(3):171.

13. Radulescu AV, Radulescu I. Rheological models for lithium and calcium greases. Mechanics. 2016;59(3):67.

14. Singh J, Kumar D, Tandon N. Development of nanocomposite grease: microstructure, flow, and tribological studies. J Tribol 2017;139(5):052001.

15. Cao ZF, Xia YQ, Liu LH, Feng X. Study on the conductive and tribological properties of copper sliding electrical contacts lubricated by ionic liquids. Tribology International 2019;130:27-35.

16. Wu PR, Kong YC, Ma ZS, et al. Preparation and tribological properties of novel zinc borate/MoS2 nanocomposites in grease. Journal of Alloys and Compounds 2018;740:823-829.

17. Dinh Le Cao KY, Bien-Cuong TRAN KHAC, Chinh Tam LE, et al. Friction characteristics of mechanically exfoliated and CVDgrown single-layer MoS2. Friction 2018;6(4):395-406.

18. Gu YF, Fei J, Huang JF, et al. Carbon microspheres coated with graphene oxide nanosheets as oilbased additives for tribological applications. Materials Today Communications 2020;25:101271.

19. Zhang L, Sun X, Liu X, et al. Alkyl titanate-modified graphene oxide as friction and wear reduction additives in PAO oil. ACS Omega 2021;6(5):3840-3846.

20. Fu YF, Li J, Zhang FQ, Xu K. The preparation and the friction and wear behaviours of TiO2/CNT/PI composite film. Journal of Experimental Nanoscience 2016;11(6):459-469.

21. Li Y, Liu XY, Liu DJ, Sun HW. Surface modified TiO2 nanoparticles-an effective anti-wear and antifriction additive for lubricating grease. Lubrication Research 2017;19(2):74-79.

22. Chen JG. Tribological properties of polytetrafluoroethylene, nano-titanium dioxide, and nano-silicon dioxide as additives in mixed oil-based titanium complex grease. Tribol Lett 2010;38:217-224.

23. Reinert L, Varenbery M, Mücklich F, Suárez S. Dry friction and wear of self-lubricating carbonnanotube-containing surfaces. Wear 2018;406-407:33-42. 
24. Chou R., Hernández Battez A., Cabello J.J, et al. Tribological behavior of polyalphaolefin with the addition of nickel nanoparticles. Tribology International 2010;43:2327-2332.

25. Battez AH, Viesca JL, Gonza'lez R, Blanco D, Asedegbega E, Osorio A. Friction reduction properties of CuO nanolubricant used as lubricant for a NiCrBSi coating. Wear 2010;268:325-8.

26. Parveez B, Wani M.F. Tribological behaviour of nano-zirconia reinforced iron-based self-lubricating composites for bearing applications. Tribology International 2021;159:106969.

27. Wang QY, Xi YC, Xu J, Liu S, et al. Study on properties of double-layered Ni-P-Cr composite coating prepared by the combination of electroless plating and pack cementation. Journal of Alloys and Compounds 2017;729:787-795.

28. Ying Wang, Xiaoxing Lu, Ningyi Yuan and Jianning Ding. A novel nickel-copper alternatingdeposition coating with excellent tribological and antibacterial property. Friction 2020;849:156222.

29. Song HY, Zhang JQ, Song XL, et al. Microstructure and friction properties of GNP/Ni-based superalloy composite coating by laser melting deposition. Applied Surface Science 2021; 541 : 148492.

30. Meng FS, Li Z, Ding HH, et al. Study on the preparation and tribological properties of BN@C-OA nanoadditive lubricants. Wear 2021; 474-475: 203876.

31. Cai, M, Liang, Y, Zhou, F, Liu, W. Tribological properties of novel imidazolium ionic liquids bearing benzotriazole group as the antiwear/anticorrosion additive in poly (ethylene glycol) and polyurea grease for steel/steel contacts. ACS Appl Mater Interfaces 2011;3(12):4580-4592.

32. Liu YH, Xin L, Zhang YJ, Chen YF, et al. The effect of Ni nanoparticles on the lubrication of a DLCbased solid-liquid synergetic system in all lubrication regimes. Tribol Lett 2017;65:31.

33. Zhao ZS, Zhang YS, Wang SF, Guo K, et.al. Tribological properties of oleylamine-modified nickel nanoparticles as lubricating oil additive. Mater. Res. Express 2019;6:105037.

34. Meng Y, Su FH and Chen YZ. Nickel/Multi-walled ncarbon nanotube nanocomposite synthesized in supercritical fluid as efficient lubricant additive for mineral oil. Tribology Letters 2018;66:134.

\section{Figures}

(a)

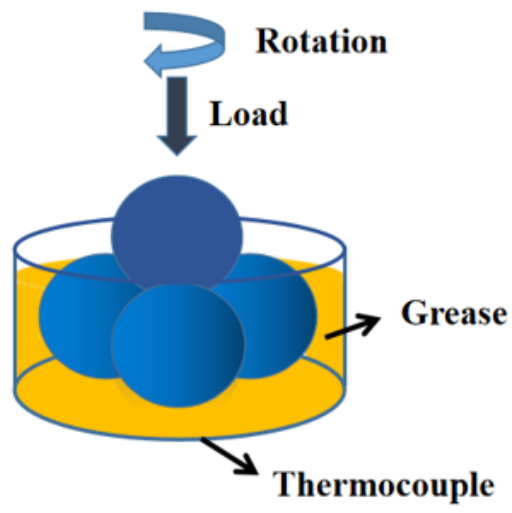

(b)

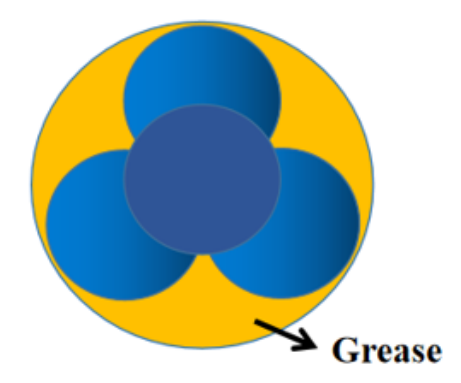

(c)

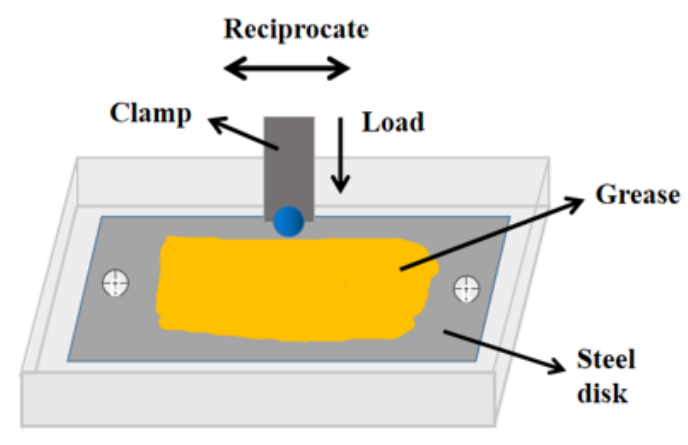


Figure 1

Schematic of four-ball test (a) front view, (b) vertical view. (c) schematic of ball-on-plate reciprocating
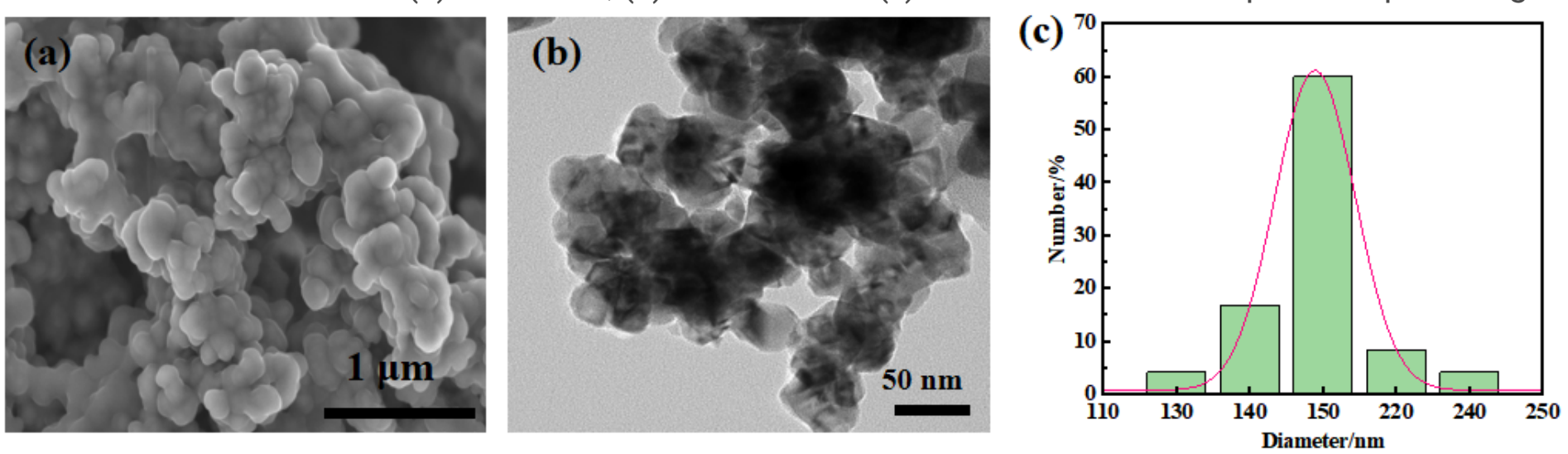

Figure 2

SEM and TEM images (a) (b) and (c) size distribution of nano-Ni
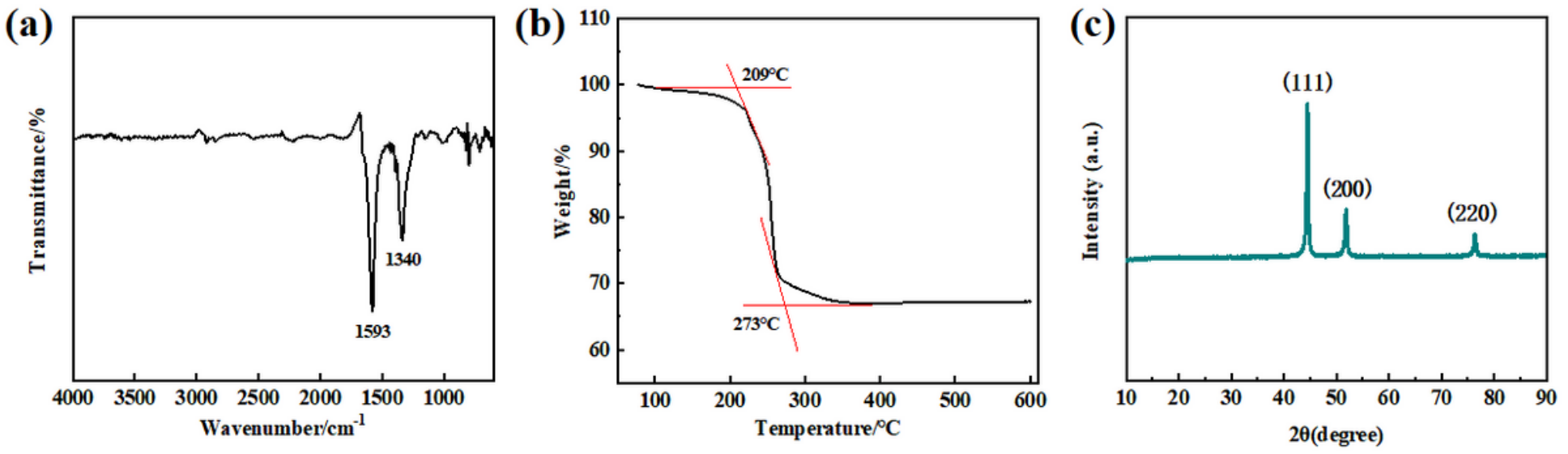

Figure 3

Characterization of nickel nanoparticles as-prepared (a) FT-IR, (b) XRD and (c) TGA. 

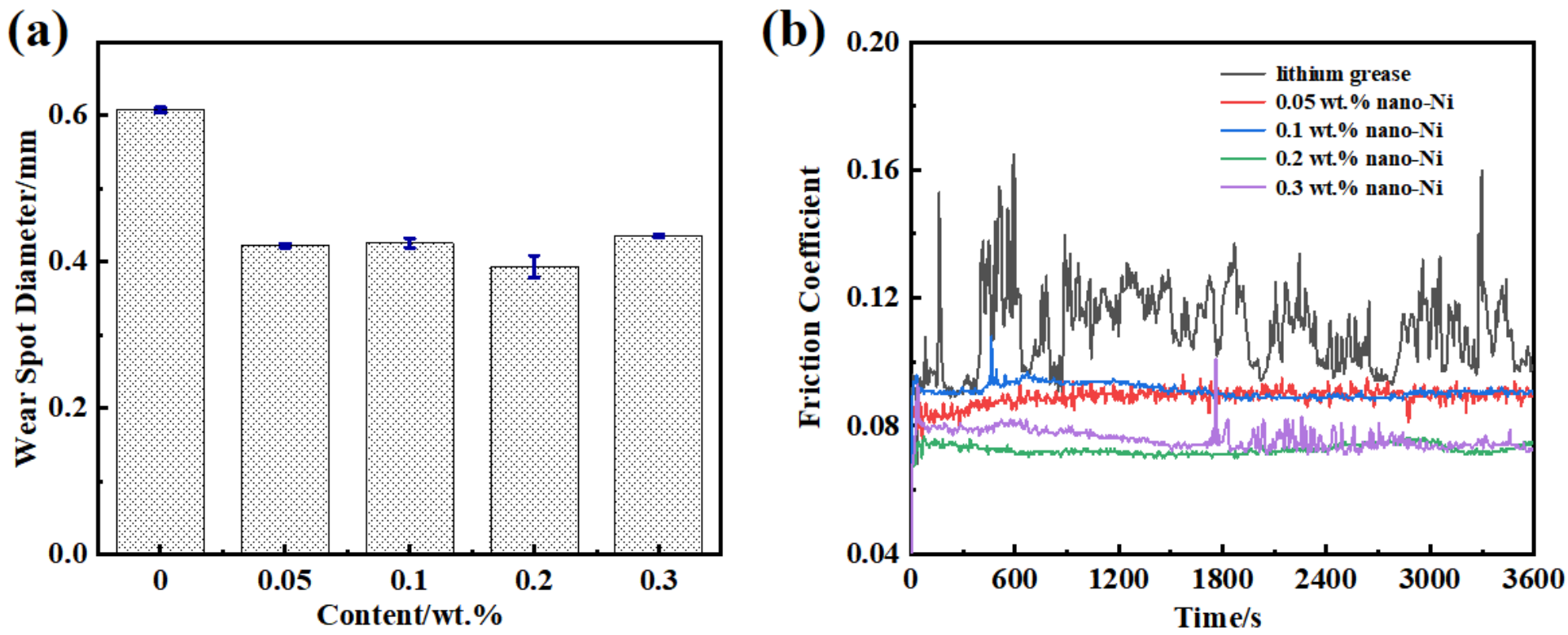

Figure 4

Comparison of the (a) average wear spot diameters (b) and the curves of friction coefficient for four-ball friction tests at $50{ }^{\circ} \mathrm{C}$.
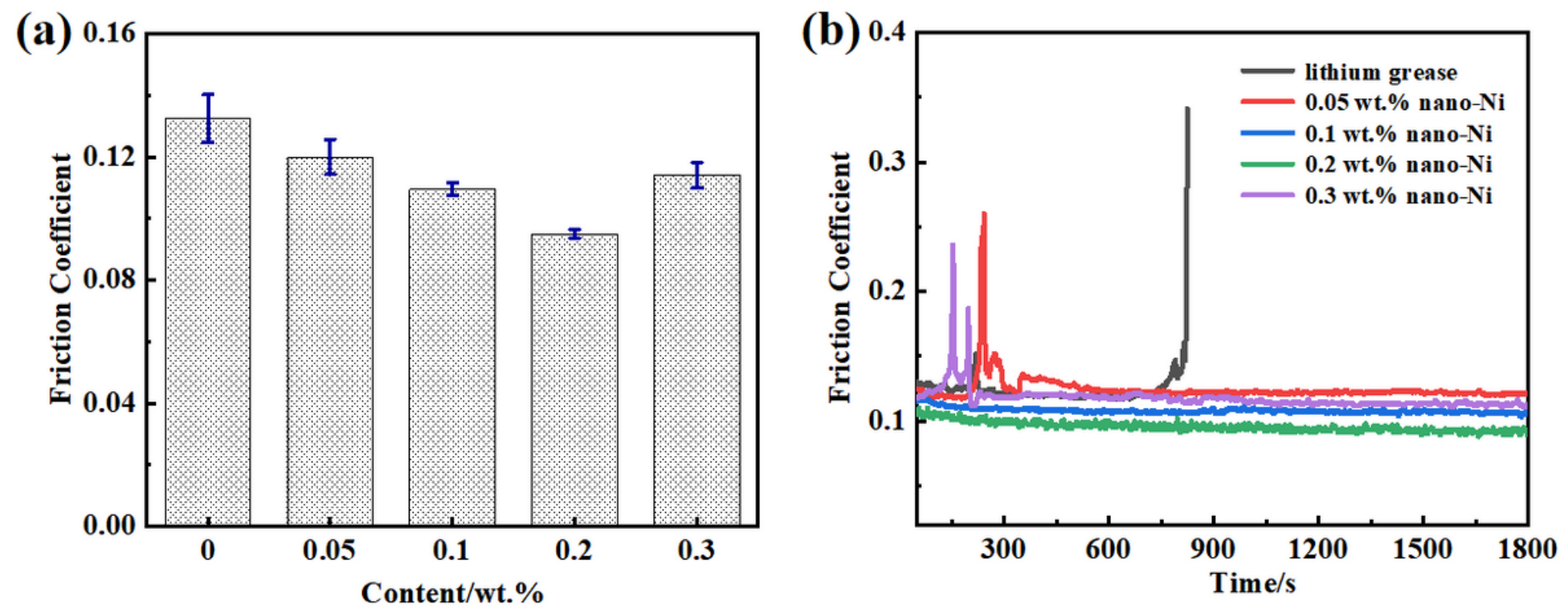

Figure 5

Comparison of the (a) average friction coefficient (b) and the curves of friction coefficient for point on flat friction tests under $40 \mathrm{~N}$ at $50{ }^{\circ} \mathrm{C}$. 

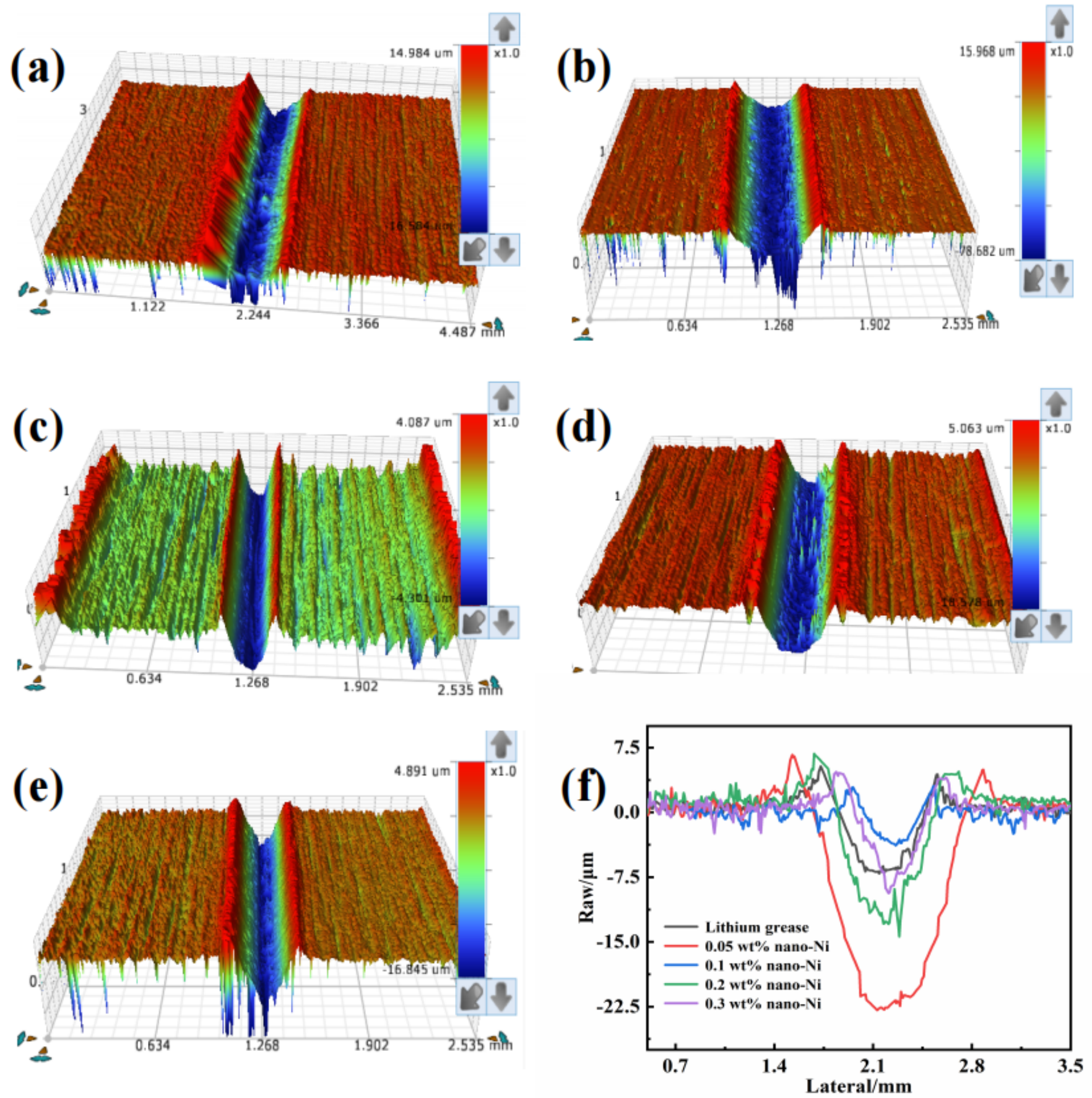

Figure 6

Wear marks photographed by WLI of different contents of nickel nanoparticlas additives. (a) lithium grease, (b) 0.05 wt\% nano-Ni, (c) 0.1 wt\% nano-Ni, (d) 0.2 wt\% nano-Ni, (e) 0.3 wt\% nano-Ni, (f) crosssectional curves of wear marks. 

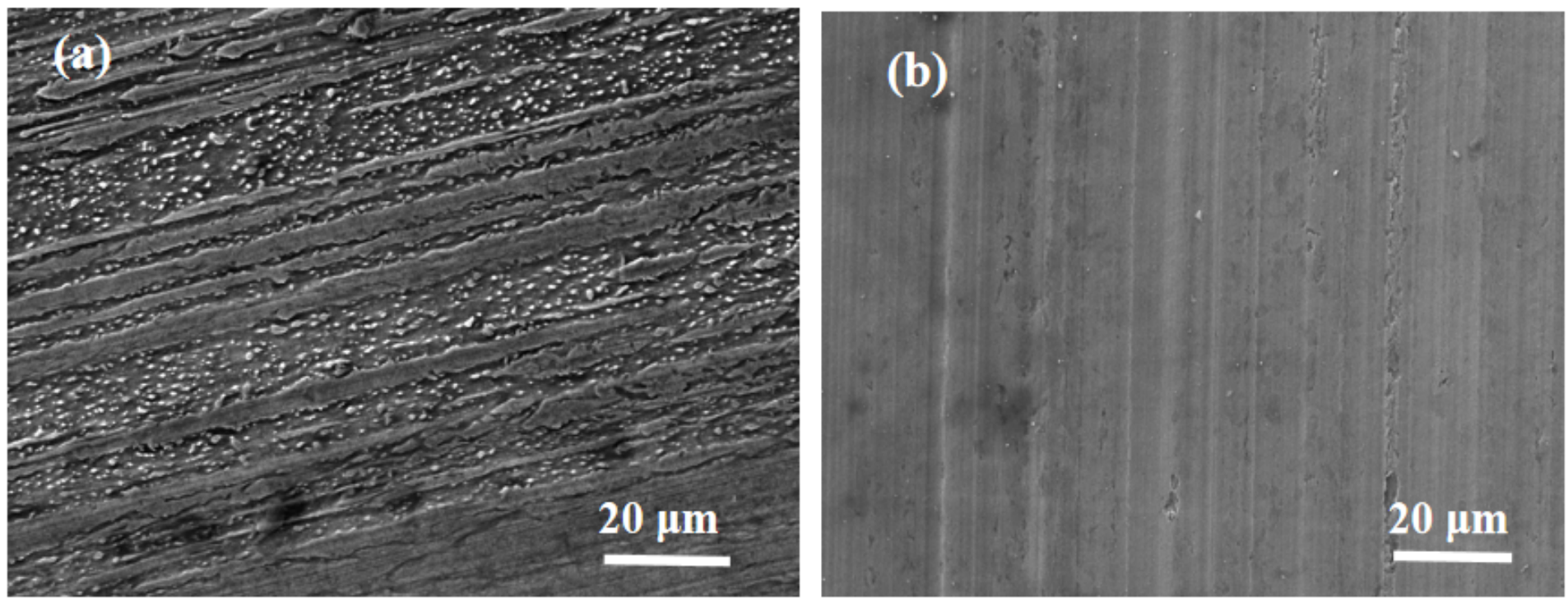

\section{Figure 7}

Morphologies of the abrasion surface of steel balls after four-ball friction tests (a) lithium grease, (b) 0.2 wt.\% nano-Ni with lithium grease
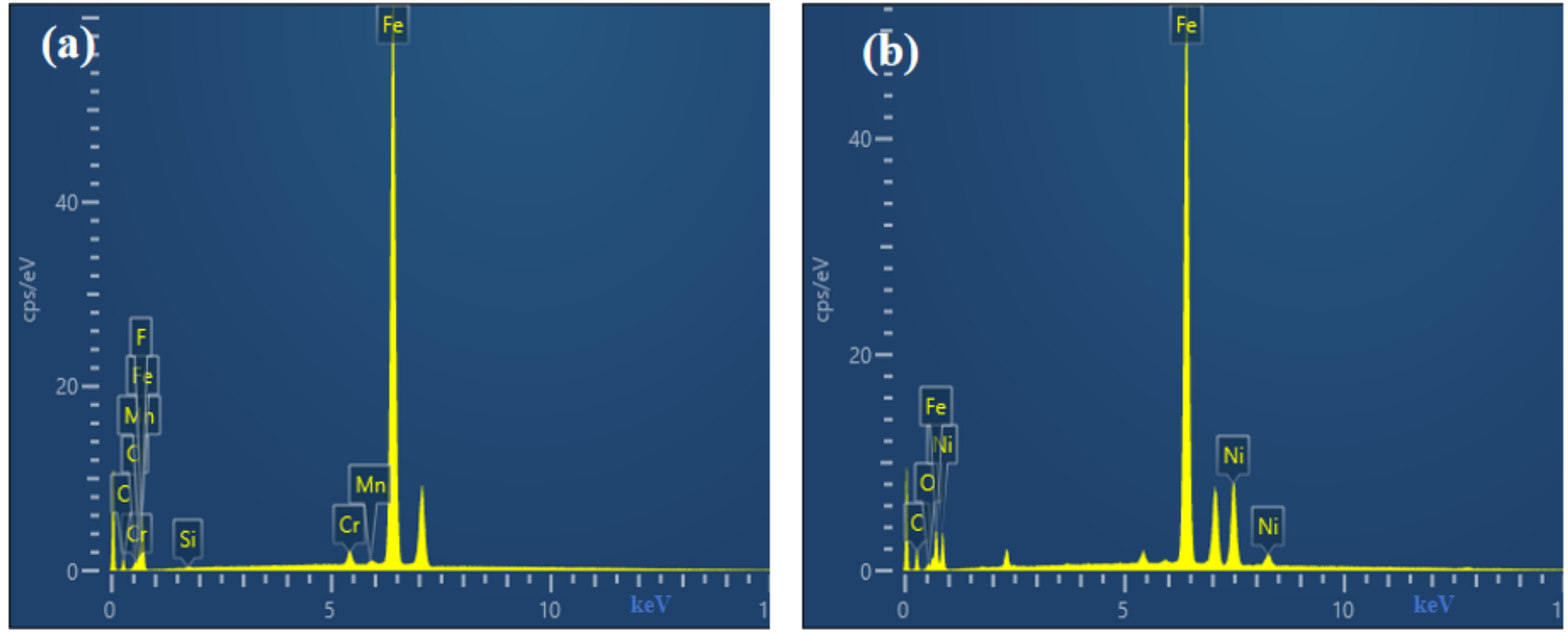

\section{Figure 8}

Comparison of the EDS energy spectra of the deposits at the grinding spots. (a) lithium grease; (b) 0.2 wt.\% nano-Ni with lithium grease 
(A)
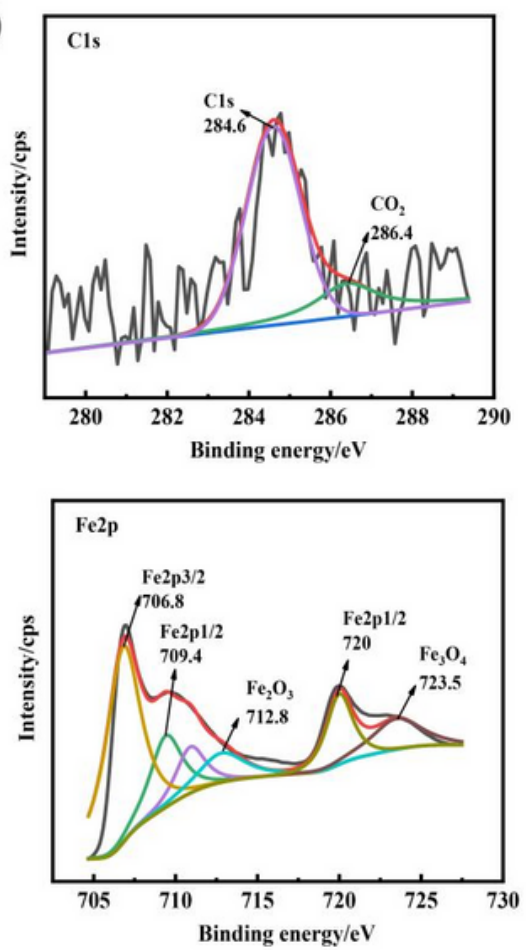

(B)
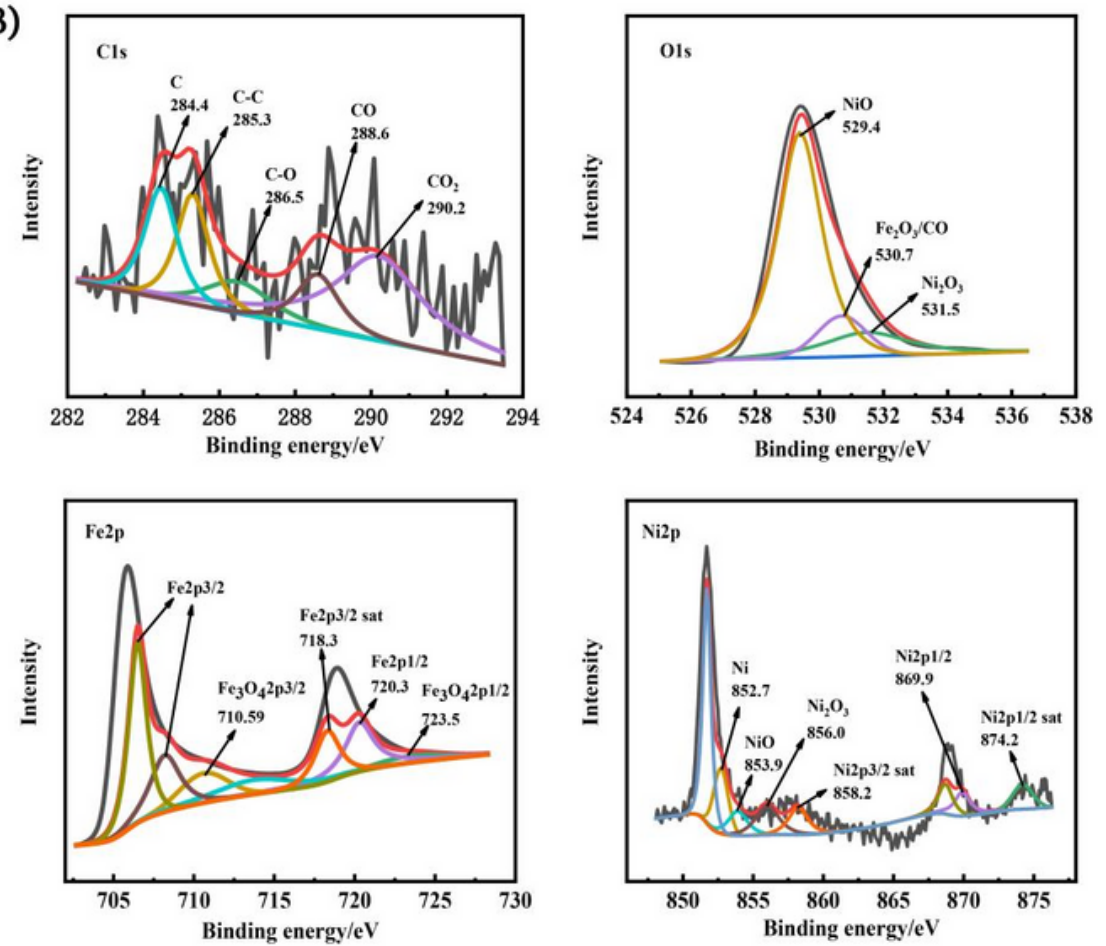

\section{Figure 9}

Comparison high-resolution XPS energy spectra C1s, 01s, Fe2p, Ni2p on the surface of the abrasion marks. (A) lithium grease; (B) $0.2 \mathrm{wt} . \%$ nano-Ni as additives in lithium grease. 
(a)

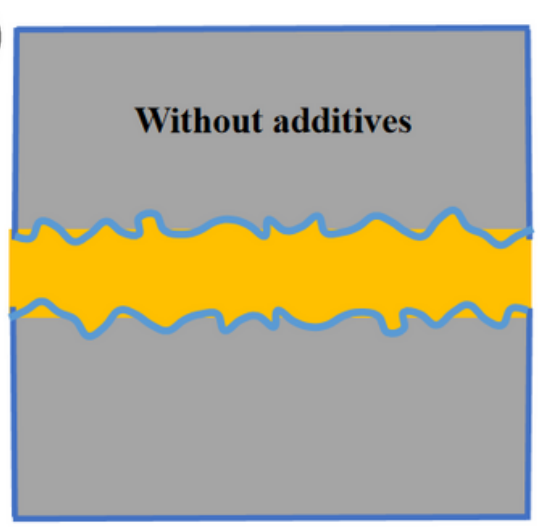

(b)

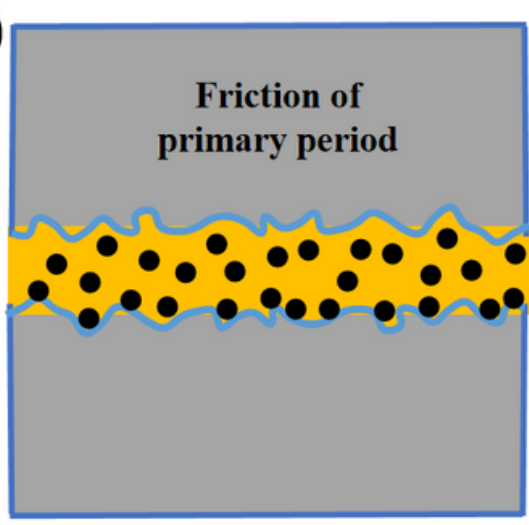

(c)

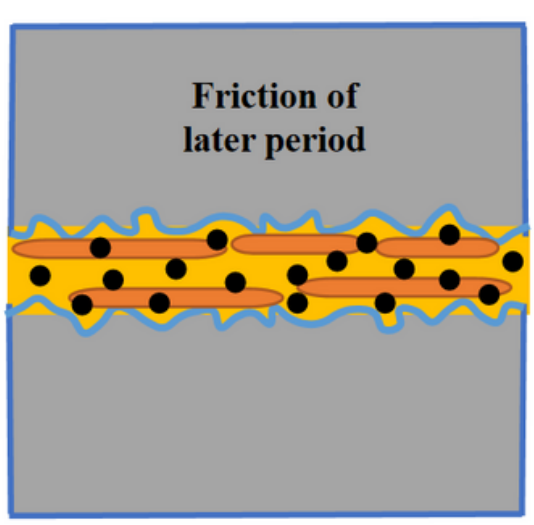

\section{Lithium grease}

\section{Chemical reaction film}

Nickel nanoparticles

Figure 10

Simulation of friction mechanism of lubricating lithium grease containing nickel nanoparticles.

\section{Supplementary Files}

This is a list of supplementary files associated with this preprint. Click to download.

- GraphicalAbstract.jpg 